\title{
Saturation in the principal (excited) channel of four-level medium as an effective two-level configuration
}

\author{
JIHAD S. ADDASI
}

Department of Applied Physics, College of Science, Tafila Technical University, 66110, Tafila, Jordan; jaddasi@edu.ttu.jo

\begin{abstract}
Saturation of complex molecules affects the nonlinear processes occurring in the medium. The medium can be described by two-, three-, and four-level configurations. The principal singlet-singlet and excited triplet-triplet channels of a four-level, N-type, configuration were used to study a nonlinear medium, which was excited by radiation with two frequencies. Under weak population of energy levels in the triplet-triplet channel (a small ratio of the total probability of spontaneous and irradiate transitions for both channels $p_{23} / p_{31}$ ), the principal (singlet-singlet) channel is similar to a two-level configuration. At the same time, the effective population of the energy levels in the triplet-triplet channel (the ratio $p_{23} / p_{31}$ is large and the radiation intensity in the principal channel is high) makes the excited (triplet-triplet) channel coincide with the two-level configuration. The saturation intensity of radiation in principal (excited) channel, as effective two-level configuration, and nonlinear processes in this channel can be controlled by external radiation acting on excited (principal) channel. Finally, the average excitation of molecules into the energy levels in the triplet -triplet channel (ratio $p_{23} / p_{31} \approx 1$ ) makes the four-level configuration similar to the three-level configuration with effective level as a result of combination between second singlet level $S_{2}$ and first triplet level $T_{1}$.
\end{abstract}

Keywords: optical properties, saturation, four-level, effective two-level.

\section{Introduction}

The nonlinear optical phenomena occur when the optical fields associated with one or more laser beams propagating in a material are large enough to create polarization fields proportional to the product of two or more of incident fields. Depending on the electronic structure of the system, excited states and multiphoton absorption, under the action of high-intensity laser radiation, can cause various nonlinear phenomena [1]. The energy structure of many organic dyes may be represented by a two-, three-, and four-level models [2-4]. Optical bistability in a two-level atomic system can be controlled by adjusting the intensity of the pump field and the detuning between two fields, while the use of the pump field reduces the threshold of optical bistability [2]]. A light -induced coherent population oscillations and ground-state Zeeman coherence in an atomic medium with degenerate two-level transitions can modify spectra of the 
applied continuous wave resonance radiation at a power level of less than $\mathrm{mW}$. The ground-state Zeeman coherence contributes to nonlinear wave mixing, while coherent population oscillations are responsible for the phase and amplitude modulation of the applied fields [ $\underline{5}]$.

Saturated absorption in three-level systems can occur in the form of two-stage transitions as a result of molecules' accumulation in the second excited state. Saturated absorption is important in nonlinear spectroscopy of complex molecules (dye solutions) and for studying absorption from excited singlet states [ $\underline{3}]$. Third order nonlinear susceptibility is derived for a three-level quantum dot system, for study: total absorption (linear and nonlinear) in GaAsP three-level quantum dot systems, four-wave mixing (FWM) for which the interplay of four coexisting four-wave mixing signals (FWMS) and amplification suppression of a degenerate FWM process in a V-type three-level atomic system were experimentally achieved $[\underline{4}, \underline{6}, \underline{7}]$. The Runge-Kutta method was used to numerically solve the rate equation of a quasi-three-level passive $Q$-switched laser. This method can be widely used for the theoretical description and optimization of a quasi-three-level passive $Q$-switched laser []ㅡ. A three-level atomic system in the radiation limit was used to control the propagation of light from a subliminal regime to a superliminal one, this scheme allows switching from one regime to another by changing the Rabi frequencies of the driving fields [9].

An open four-level system having two pairs of closely spaced levels (N-type configuration) is controlled by a single electromagnetic field and tuned to resonance with an average frequency of four allowed dipole transitions. Analytical expressions for effective line widths, effective detuning and induced polarization clearly indicate the role of quantum interference [10].

Effective two-level models have been widely used to describe squeezing effects in two-photon bistability in ladder three-level configurations. An effective two-level model was derived from the full three-level model, which considered the assumption of large detuning of the atomic transition and the adiabatic elimination of the variables of the intermediate level. The comparison between the effective two-level and full three-level models at a semiclassical and fully quantum levels was discussed [11]. The system of a three-level atom interacting with two quantized modes in the $\Xi$ configuration with arbitrary detuning can be exactly reduced to an effective two-level system with an effective Raman coupling, which depends nonlinearly on the intensity of the two cavity fields. A Hamiltonian was obtained in which one of the three levels is decoupled and the effective coupling does not show any singularity for any rations of coupling parameters [12]. The dynamics of the parity- and time-reversal symmetric two-level atoms in three regimes was studied, unbroken, broken symmetries and exception point. The dynamics of an effective model with balanced gain and loss was investigated. Moreover, the effectively conserved two-level Hamiltonian is derived from the $\Lambda$-type model coupled with two optical fields and one radio-frequency field by means of adiabatically eliminating the third energy level [1]ㅡ.

The two-level system is not only the key element in various fields of contemporary physics, such as radiation-matter interactions and collision physics, but also the funda- 
mental building block of modern applications ranging from quantum control to quantum information processing. A simple analytical solution of a two-level atom interacting with a polychromatic driving field was discussed. A polychromatic driving field consists of a central field with frequency $\omega$ and $N$ pairs of symmetrically fields with other frequencies, which dispersively drive the transitions among the states of the two-level atom. This model was generalized to a $\Lambda$ type atom interacting with a polychromatic driving field. Besides the analogous results to the two-level atom, the robustness of the three-level system against atomic spontaneous emission was improved [14]. A highly efficient inner-state enantioseparation was achieved based on the cyclic three-level systems of chiral molecules. By choosing the appropriate parameters under the three -photon resonance condition, the two enantiomers initially prepared in their corresponding ground states can be governed by chirality-dependent effective two-level models. The corresponding effective two-level models have the same effective Rabi frequencies but different effective detunings [15].

The three-level and four-level atomic configurations under influence of radiation with two frequencies were used to study the nonlinear optical properties of nonlinear media. These theoretical studies were carried out for media with coincident emission and absorption bands and for media with Stokes shifted emission bands. The tuning frequency of various radiation and the bleaching conditions for dye solutions were taken into account and compared in some cases with a two-level model. In addition, for these configurations, the possibility of controlling nonlinear effects in a given channel (principal or excited) by radiation in another channel was studied [ $\underline{16}-\underline{20}]$.

The aim of this work is to study the saturation intensity of radiation in both principal (singlet-singlet) and excited (triplet-triplet) channels in four-level configuration, also to find conditions necessary for these channels to be comparable with the two-level and three-level model. The saturation intensity of radiation in the principal and excited channels in a four-level medium (with an N-type) will be studied in the cases of effective and weak transitions of molecules into the excited triplet-triplet channel. In addition, the saturation intensity of radiation in principal (excited) channel is effectively controlled by external radiation in another channel. This control of nonlinear processes could be achieved by noncoherent radiation in one channel with a certain frequency. This radiation effectively acts on nonlinear processes that happened in other channel due to high coherent radiation with different frequency.

\section{Theory and analysis}

The saturation intensity of radiation for a four-level medium under the action of radiation with two frequencies $\left(\omega_{0}, \omega\right)$ can be written as follows:

$$
I_{12}^{\mathrm{sat}}=\frac{1+a I_{34}}{J+b I_{34}}
$$

for radiation in the principal (singlet-singlet $S_{0}-S_{1}$ ) channel, and 


$$
I_{34}^{\mathrm{sat}}=\frac{1+J I_{12}}{a+b I_{12}}
$$

for radiation in the excited (triplet-triplet $T_{1}-T_{2}$ ) channel [15], where:

$$
\begin{aligned}
& J=\frac{B_{12}\left(1+p_{23} / p_{31}\right)+B_{21}}{v\left(p_{21}+p_{23}\right)} \\
& a=\frac{B_{43}}{v p_{43}} \\
& b=a J+\frac{\left(p_{23} / p_{31}\right) B_{12} B_{34}}{v^{2}\left(p_{21}+p_{23}\right) p_{43}}
\end{aligned}
$$

and $p_{i j}$ is the total probability of spontaneous and irradiation transitions in the $i-j$ channel, the Einstein coefficients $B_{12}\left(\omega_{0}\right)$ and $B_{21}\left(\omega_{0}\right)$ are determined at radiation frequency $\omega_{0}$ in the principal singlet-singlet channel, and $B_{34}(\omega), B_{43}(\omega)$ are determined at the radiation frequency $\omega$ in the excited triplet-triplet channel [19].

The radiation intensity $I_{12}^{\text {sat }}$ in the principal channel $\left(S_{1}-S_{2}\right)$ and radiation intensity $I_{23}^{\text {sat }}$ in excited $\left(S_{2}-S_{3}\right)$ channel for three-level configuration can be written in the following form:

$$
\begin{aligned}
& I_{12}^{\text {sat }}=\frac{1+a I_{23}}{J+b I_{23}} \\
& I_{23}^{\text {sat }}=\frac{1+J I_{12}}{a+b I_{12}}
\end{aligned}
$$

where $[\underline{17}, \underline{18}]$ :

$$
\begin{aligned}
& J=\frac{B_{12}+B_{21}}{v p_{21}} \\
& a=\frac{B_{32}}{v p_{32}} \\
& b=a J+\frac{B_{12} B_{23}}{v^{2} p_{21} p_{32}}
\end{aligned}
$$

The saturation intensity $I_{\mathrm{s}}$ for a two-level model is determined by the following equation:

$$
I_{\mathrm{s}}=1 / J
$$

where $J=\left(B_{12}+\mathrm{B}_{21}\right) /\left(v p_{21}\right)[\underline{17}, \underline{18}]$. 
Now let's look at a four-level configuration and compare the principal and excited channels of this configuration with a two-level configuration, and compare the four-level configuration with the three-level model with effective level as a result of combination between second singlet level $S_{2}$ and first triplet level $T_{1}$. Bleaching of the medium in the principal or excited channels depends not only on the radiation intensity in another channel, but also on the ratio of the total probability of spontaneous and irradiation transitions $p_{23} / p_{31}$ for both the principal and excited channels.

The numerical calculations in this paper will be taken for a nonlinear medium with a Gaussian shape $\left(B_{i j}=B_{j i}=B_{i j}^{\max } \exp \left(-\eta_{i j}^{2}\right)\right)$ of coincident absorption and emission bands ( $\omega_{j i}=\omega_{i j}$, and $\omega_{i j}$ is the frequency of the centre of $i-j$ absorption (emission) band) with a profile of halfwidth $\Delta_{i j}$ at $1 / \mathrm{e}$ height for $i-j$ band. The frequency tuning of the radiation in the principal $\left(\eta_{12}=\left(\omega_{0}-\omega_{12}\right) / \Delta_{12}\right.$, and $\left.\eta_{21}=\left(\omega_{0}-\omega_{21}\right) / \Delta_{12}\right)$ and in the excited $\left(\eta_{34}=\left(\omega-\omega_{34}\right) / \Delta_{34}\right.$, and $\left.\eta_{43}=\left(\omega-\omega_{43}\right) / \Delta_{34}\right)$ channels equals to zero, which means that the radiation frequencies are tuned into the centre of the absorption and emission bands for both the principal and excited channels. The intensities $I_{12}$ and $I_{34}$ of the radiation acting in the principal and excited channels are normalized to the values $v\left(p_{21}+p_{23}\right) / B_{12}^{\max }$ and $v p_{43} / B_{34}^{\max }$, respectively.

Equations (1)-(5) are illustrated in Fig. 1. Figure 1 and Eqs. (1) and (2) show that the saturation intensities: $I_{12}^{\text {sat }}$ in the principal (Fig. 1a) and $I_{34}^{\text {sat }}$ in the excited channels (Fig. 1b) decrease with increasing ratio of the total probability of spontaneous and irradiation transitions $p_{23} / p_{31}$ and decrease with increasing radiation intensity in another channel.

The saturation intensities in the principal and in the excited channels significantly depend on the ratio $p_{23} / p_{31}$ and the four-level configuration itself also significantly depends on this ratio. Let's consider three cases:

The first case. Small transitions of molecules from the singlet-singlet channel to the triplet-triplet channel for a small ratio $p_{23} / p_{31} \approx 0$.
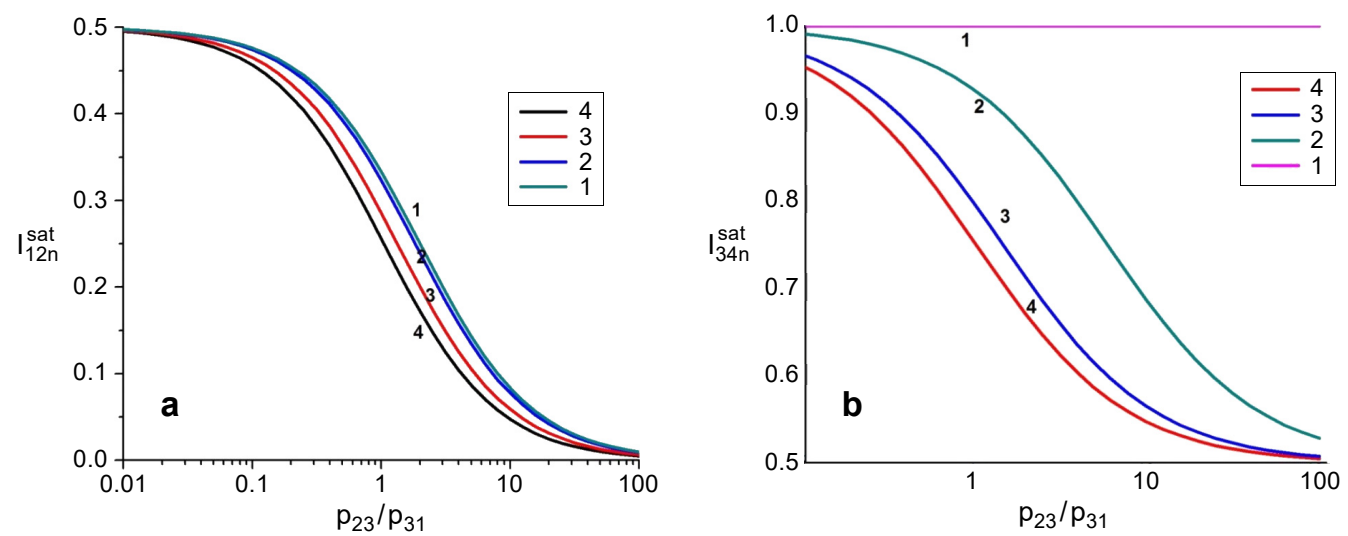

Fig. 1. Dependence of the saturation intensity: (a) in the principal channel $I_{12 n}^{\text {sat }}$, (b) in the excited channel $I_{34 n}^{\text {sat }}$ on ratio $p_{23} / p_{31}$ for frequency tuning of radiation: in the principal and the excited channels $\eta_{12}=\eta_{34}=0$. Curves $1,2,3,4$ at: (a) $I_{34 n}=0,0.1,1,10$, and (b) $I_{12 n}=0,0.1,1,10$, respectively. 

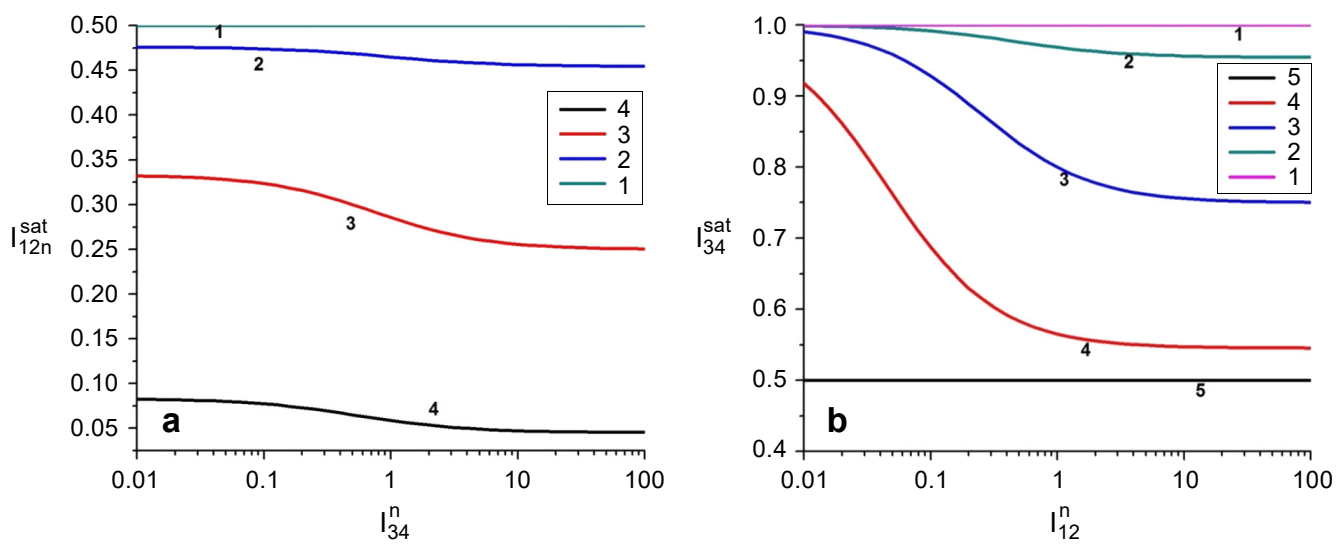

Fig. 2. Dependence of saturation intensity: (a) in the principal channel $I_{12 n}^{\text {sat }}$ on the radiation intensity in the excited channel $I_{34}^{n},(\mathbf{b})$ in the excited channel $I_{34 n}^{\text {sat }}$ on the radiation intensity in the principal channel $I_{12}^{n}$ for frequency tuning of radiation: in the principal and excited channels $\eta_{12}=\eta_{34}=0$. Curves 1 , 2,3 and 4 at ratio $p_{23} / p_{31}=0,0.1,1$ and 10 . Curve 1 (a) and curve 5 (b) are for two-level configuration.

For this case we can write: $J \approx\left\{B_{12}+B_{21}\right\} /\left(v\left(p_{21}+p_{23}\right)\right), a=B_{43} /\left(v p_{43}\right), b \approx a J$, $I_{12}^{\text {sat }} \approx 1 / J$ for any intensity $I_{34}$, and $I_{34}^{\text {sat }} \approx 1 / a$ for any intensity $I_{12}$.

The principal (singlet-singlet) channel is consistent with the two-level model, see curve 1, Fig. 2a. In this case, the number of molecules at the lower singlet energy level is very large, and the transitions from the lower singlet energy level $S_{1}$ to the upper singlet energy level $S_{2}$ and from the upper to lower energy levels affect the value of the saturation intensity $\left(B_{12}+B_{21}\right)$. In addition, the saturation intensity $I_{34}^{\text {sat }}$ in the triplet -triplet channel is independent of the radiation intensity in singlet-singlet channel $\left(I_{12}\right)$, which is determined by transitions from the upper triplet energy level to the lower triplet energy level only in this channel due to the small number of molecules in lower triplet energy level $\left(I_{34}^{\text {sat }}=1 / a, a=B_{43} /\left(v p_{43}\right)\right)$, see curve 1 , Fig. $2 \mathbf{b}$.

The second case. Effective transitions of molecules from singlet-singlet to triplet -triplet channels $p_{23} / p_{31} \gg 1$ and $I_{12} \gg 1$.

For this case, we can write:

$$
\begin{aligned}
& J=\frac{p_{23}}{p_{31}} \frac{B_{12}}{v\left(p_{21}+p_{23}\right)} \\
& a=\frac{B_{43}}{v p_{43}} \\
& b=J\left(a+\frac{B_{34}}{v p_{43}}\right) \\
& I_{12}^{\text {sat }}=\frac{1+a I_{34}}{J+b I_{34}}
\end{aligned}
$$


as in Eq. (1) and for $p_{23} / p_{31} \gg 1$ the saturation intensity in the excited channel is

$$
I_{34}^{\mathrm{sat}}=\frac{1+J I_{12}}{a+J\left(\left(B_{34}+B_{43}\right) /\left(v p_{43}\right)\right) I_{12}}
$$

and for effective transitions of molecules into triplet-triplet channel $I_{12} \gg 1$, the saturation intensity in the excited channel will be

$$
I_{34}^{\mathrm{sat}}=\frac{1}{\left(B_{34}+B_{43}\right) /\left(v p_{43}\right)}
$$

which is of the same form as two-level model, see Eq. (5).

Figure 2 a shows that the saturation intensity $I_{12}^{\text {sat }}$ in the principal channel is lower than for two-level model (curve 1) and for a large ratio $p_{23} / p_{31}$, the bleaching is more simple. For the excited channel, the saturation intensity $I_{34}^{\text {sat }}$ is determined by the transitions of molecules from the upper energy level to the lower energy level for small pumping of molecules into this channel (small $I_{12}$ ), Fig. 2 b.

At the same time, for effective pumping of molecules into the triple-triplet channel $\left(I_{12} \gg 1, p_{23} / p_{31} \gg 1\right)$, the number of molecules at the lower triplet energy level $T_{1}$ is large. For high pumping of molecules into triplet lower energy level $T_{1}$, the saturation intensity $I_{34}^{\text {sat }}$ is determined by transitions from the lower to the upper and from upper to lower energy levels, which leads to small value of saturation intensity, see curve 4, Fig. 2 b.

The third case. An effective interaction between molecules in both singlet-singlet and triplet-triplet channels $p_{23} / p_{31} \approx 1$.

For this case we can write:

$$
\begin{aligned}
& J=\frac{2 B_{12}+B_{21}}{v\left(p_{21}+p_{23}\right)} \\
& a=\frac{B_{43}}{v p_{43}} \\
& b=a J+\frac{B_{12} B_{34}}{v^{2}\left(p_{21}+p_{23}\right) p_{43}}
\end{aligned}
$$

which is not much different from the three-level configuration, see Eqs. (1) and (2). Figure 3 shows that the saturation intensities in the principal (excited) channel are slightly higher for the four-level configuration (curves 1 and 2) than for the three-level model Eqs. (3) and (4) (curve 6) for a small ratio $p_{23} / p_{31}$ and a small radiation intensity in another channel.

In addition, Fig. 3 shows that the saturation intensities in the principal (excited) channel are slightly lower for the four-level configuration (curves 4,5 ) than for the 

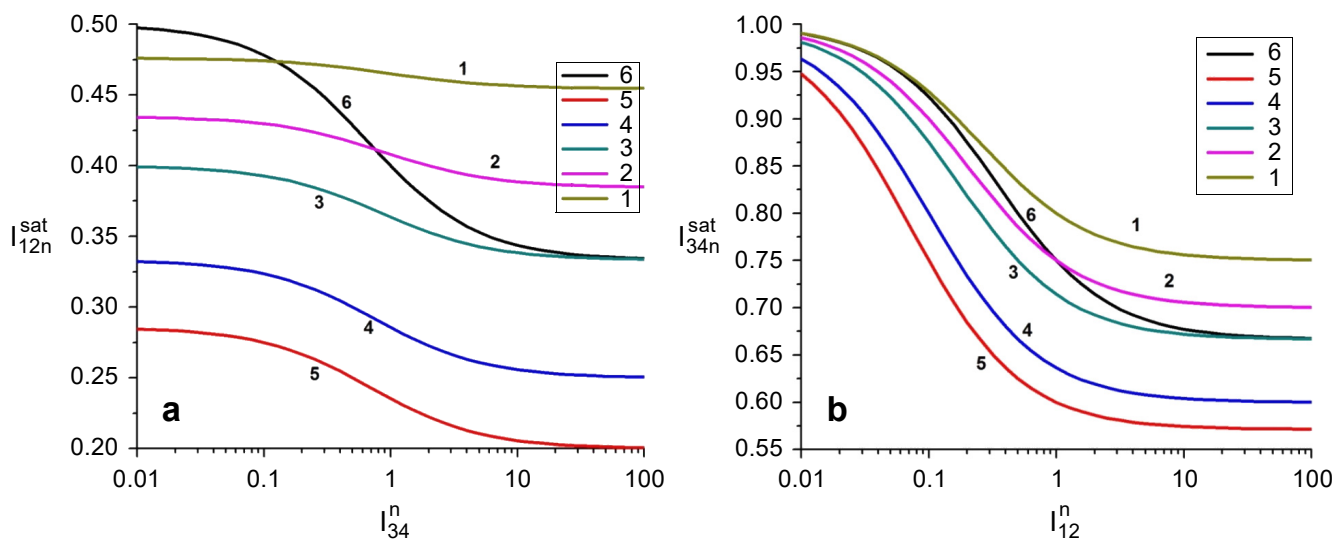

Fig. 3. Dependence of the saturation intensity: (a) in the principal channel $I_{12 n}^{\text {sat }}$ on the radiation intensity in the excited channel $I_{34}^{n}$, (b) in the excited channel $I_{34 n}^{\text {sat }}$ on the radiation intensity in the principal channel $I_{12}^{n}$ for frequency tuning of radiation: in the principal and excited channels $\eta_{12}=\eta_{34}=0$. Curves 1 , $2,3,4$ and 5 at ratio (a) $p_{23} / p_{31}=0.1,0.3,0.5,1$ and 1.5 , (b) $p_{23} / p_{31}=1,1.5,2,4$ and 6 . Curve 6 is for three-level configuration.

three-level model (curve 6) for a slightly large ratio $p_{23} / p_{31}$ and high radiation intensity in another channel. All results illustrated in Figs. 1-3, show that by changing the incoherent radiation in a particular channel, it is possible to control any nonlinear processes, such as: four-wave mixing, bistability, and holography, achieved in another channel spatially excitation of molecules can be done under the action of radiation with two frequencies.

\section{Conclusion}

For a nonlinear medium with coincident absorption and emission bands modelled by a four-level configuration, the saturation intensity in the principal (singlet-singlet) channel $I_{12}^{\text {sat }}$ (in the excited triplet-triplet channel $I_{34}^{\text {sat }}$ ) decreases with increasing ratio of the total probability of spontaneous and irradiation transitions $p_{23} / p_{31}$. For a small ratio $p_{23} / p_{31}$, weak transitions of molecules to an excited triplet-triplet channel, and the principal (singlet-singlet) channel are very similar to a two-level model, and the saturation of nonlinear processes in this channel is easier for high radiation intensity in the excited triplet-triplet channel. In addition, a large ratio $p_{23} / p_{31}$ and a large effective pump of molecules $\left(I_{12} \gg 1\right)$ to the excited channel make the excited (triplet -triplet) channel similar to a two-level model and saturation of nonlinear processes in this channel easier with a high radiation intensity in the principal (singlet-singlet) channel. Finally, for the ratio $p_{23} / p_{31} \approx 1$, four-level model is similar to a three-level configuration with an effective level as a result of combination between second singlet level $S_{2}$ and first triplet level $T_{1}$. 


\section{References}

[1] NiE W., Optical nonlinearity: phenomena, applications, and materials, Advanced Materials 5(7-8), 1993, pp. 520-545, DOI: 10.1002/adma.19930050704.

[2] Mahmoudi M., Sahrai M., Mousavi S.M., Controlling the optical bistability and multistability in a two-level pumped-probe system, Journal of Luminescence 130(5), 2010, pp. 877-881, DOI: 10.1016/j.jlumin.2009.12.018.

[3] Bezrodny V.I., Przhonskaya O.V., Tikhonov E.A., Shpak M.T., Saturated absorption as a result of two-stage transitions in dye solutions, Kvantovaya Elektronika 8, 1981, pp. 410-412.

[4] Al-Khursan A.H., Al-KhaKani M.K., Al-Mossawi K.H., Third-order non-linear susceptibility in a three-level QD system, Photonics and Nanostructures: Fundamentals and Applications 7(3), 2009, pp. 153-160, DOI: 10.1016/j.photonics.2009.06.004.

[5] Akulshin A.M., McLean R.J., Sidorov A.I., Hannaford P., Probing degenerate two-level atomic media by coherent optical heterodyning, Journal of Physics B: Atomic, Molecular and Optical Physics 44(17), 2011, article 175502, DOI: 10.1088/0953-4075/44/17/175502.

[6] Asadpour S.H., Sahrai M., Soltani A., Hamedi H.R., Enhanced Kerr nonlinearity via quantum interference from spontaneous emission, Physics Letters A 376(3), 2012, pp. 147-152, DOI: 10.1016/ j.physleta.2011.11.011.

[7] Zaho Y., Li Y., Wang Z., Zhao Z., Li N., Zheng H., Sang S., Zhang D., Zhang Y., Polarization dressings of four-wave mixing process in a $V$-type three-level atomic system, Optics Communications 285(6), 2012, pp. 1397-1403, DOI: 10.1016/j.optcom.2011.09.072.

[8] Li Q., Feng B., Zhang Z., Zhang T., Direct numerical simulation of quasi-three-level passive $Q$-switched laser, Optics Communications 284(13), 2011, pp. 3391-3398, DOI: 10.1016/j.optcom. 2011.03.061.

[9] Gonzalo I., Anton M.A., Carreno F., Calderon O.G., Subluminal and superluminal propagation in a three-level atom in the radiative limit based on coherent population oscillations, Physics Letters A 372(42), 2008, pp. 6334-6339, DOI: 10.1016/j.physleta.2008.09.002.

[10] Ghosh S., Mandal S., An analytical study on absorptive lineshape of a driven N-type open four -level system: quantum interference effects, Optics Communications 283(9), 2010, pp. 1832-1839, DOI: 10.1016/j.optcom.2010.01.006.

[11] Sinatra A., Gastelli F., Lugiato L.A., Grangier P., Poizat J.P., Effective two-level model versus three level model, Quantum and Semiclassical Optics 7(3), 1995, pp. 405-421, DOI: 10.1088/1355-5111/ 7/3/016.

[12] WU Y., YANG X., Effective two-level model for a three-level atom in the $\Xi$ configuration, Physical Review A 56(3), 1997, pp. 2443-2446, DOI: 10.1103/PhysRevA.56.2443.

[13] Du L., Xu Z., YIn C., Guo L., Dynamical evolution of an effective two-level system with PT symmetry, Chinese Physics Letters 35(5), 2018, article 050301, DOI: 10.1088/0256-307X/35/5/050301.

[14] Li D.X., Shaо X.Q., Rapid population transfer of a two-level system by a polychromatic driving field, Scientific Reports 9, 2019, article 9023, DOI: 10.1038/s41598-019-45558-5.

[15] Ye C., Zhang Q., Chen Y.-Y., Li Y., Effective two-level models for highly efficient inner-state enantioseparation based on cyclic three-level systems of chiral molecules, Physical Review A 100(4), 2019, article 043403, DOI: 10.1103/PhysRevA.100.043403.

[16] Addasi J.S., Saturation in principal channel for nonlinear media with Stokes shifting emission bands, Journal of Applied Sciences 5(8), 2005, pp. 1484-1488, DOI: 10.3923/jas.2005.1484.1488.

[17] Addasi J.S., Jadan M., Abushendi S.A., Saturation processes in principal channel of dye solutions with coincident absorption and emission bands - part II, Journal of Applied Sciences 7(22), 2007, pp. 3588-3591, DOI: 10.3923/jas.2007.3588.3591.

[18] AdDasi J.S.M., Saturation intensity in excited channel for nonlinear medium with coincident absorption and emission bands - part I, Journal of Applied Sciences 7(19), 2007, pp. 2896-2899, DOI: 10.3923/jas.2007.2896.2899. 
[19] Addasi J., Saturation processes in nonlinear media modeled by four-level configuration with coincident absorption and emission bands, Acta Physica Polonica A 122(1), 2012, pp. 156-158, DOI: 10.12693/APhysPolA.122.156.

[20] ADDASI J.S., The dependence of complex refractive index for four-level atom on radiation frequencies, Optik - International Journal for Light and Electron Optics 124(18), 2013, pp. 3250-3253, DOI: $\underline{10.1016 / \text { j.ijleo.2012.10.006. }}$.

Received March 20, 2020

in revised form May 7, 2020 